\title{
A short proof to Brauer's third main theorem
}

\author{
By Arye Juhász
}

(Received February 16, 1983; Revised July 18, 1983)

\section{Introduction}

In this note we present a short proof which uses Brauer's first main theorem, Nagao's lemma and some basic results from block thoery.

\section{Acknowledgements}

I am indebted to the referee for his useful suggestions. This work was written while I was a Feinberg Fellow in the Department of Pure Mathematics, The Weizmann Institute of Science, Rehovot. I am grateful to the Foundation for the grant and to the Department for its generous hospitality.

\section{Notation and basic results}

In this paper $G$ is a finite group of order $|G|, K$ is a field of characteristic $p>0$ which is algebraically closed (however see the remark at the end) and $K G$ is the group-algebra. Let $H$ be a subgroup of $G . C_{G}(H)$ and $N_{G}(H)$ stand for the centralizer and the normalizer of $H$ in $G$, respectively. Following R. Brauer, we shall call a block $b$ of $H$ admissible if $b$ has a defect group $D$ such that $C_{G}(D) \subseteq H$.

We recall some definitions and results for convenience.

(a) A block $b$ of $H$ is called the principal block if $b$ contains the trivial representation of $H$.

(b) The defect groups of the principal block of $H$ and the vertices of the trivial representation are Sylow $p$-subgroups of $H$.

(c) ([2, sec. 6]) If $B$ is a block of $G$ with a defect group $D$ and $D C_{G}(D)$ $\subseteq H$, then $H$ has a block $b$ with a defect group $D$ which satisfies $b^{G}=B$.

(d) ([2, sec. 6]) Let $b_{1}$ and $b_{2}$ be admissible blocks of $H$ satisfying $b_{1}^{G}=b_{2}^{G}$. If $H$ is normal in $G$ then $b_{1}$ is conjugate to $b_{2}$ in $G$.

(e) $([1,57.4,58.3])$ Let $b$ be a block of $H$ with a defect group $D$. If $b^{G}$ is defined then it has a defect group which contains $D$.

(f) Brauer's first main theorem $([1,65.4])$. Let $D$ be a $p$-subgroup of $G$ and let $H=N_{G}(D)$. There exists a one to one correspondence between the blocks of $G$ with a defect group $D$ and the blocks of $H$ with defect 
group $D$. This correspondence is given by $b \leftrightarrow b^{G}$.

(g) Nagao's lemma $([1,56.4])$. Let $Q$ be a $p$-subgroup of $G$ and assume that $H$ is a subgroup of $G$ which satisfies $Q C_{G}(Q) \subseteq H \subseteq N_{G}(Q)$. Let $M$ be a $K G$-module in a block $B$ of $G$. Then every component $V$ of $\mathrm{M}_{H}$ with a vertex $U \geqslant Q$ belongs to a block $b$ of $H$ with $b^{a}=B$.

For unexplained terms see [1].

\section{Preparatory results}

(a) LEMMA. Let $b$ be an admissible block of $H$. If $b$ is the principal block of $H$ then $b^{G}$ is the principal block $B_{0}$ if $G$.

Proof. For a subgroup $X$ of $G$, let $1_{X}$ be the trivial one dimensional representation of $X$. By 1 (a) $1_{X}$ belongs to the principal block of $X$. Let $D$. be a defect group of $b$ with $C_{G}(D) \subseteq H$. If $H \subseteq N_{G}(D)$ then 1 (g) and $1(\mathrm{~b})$ with $M=1_{G}$ and $V=1_{H}$ implies $b^{G}=B_{0}$. If $H \leftarrow N_{G}(D)$ then let $N=N_{G}(D) \cap H$ and let $\beta$ be the Brauer correspondent of $b$ in $N$. Thus $\beta^{H}=b$. (See 1). Now $1(\mathrm{~b}), 1(\mathrm{~g})$ with $M=1_{H}$ and $V=1_{N}$ implies that $\beta$ is the principal block of $N$. By the above argument $\beta^{G}=B_{0}$. But $\beta^{G}=\left(\beta^{H}\right)^{G}=b^{G}$, hence $b^{G}$ is the principal block of $G$.

(b) Lemma. Let $H$ be a normal subgroup of $G$ and $b_{0}$ the principal block of $H$. Then for every $g \in G, b_{0}^{g}=b_{0}$.

Proof. $1_{H} \bigotimes_{H} g \cong 1_{H}$ as $k H$-modules. On the other hand $1_{H} \otimes_{H} g$ belongs to $b_{0}^{g}$. Hence the result follows by 1 (a).

(c) LEMMA. Let $H$ be a subgroup of $G$ and $b$ an admissible block of $H$ with a defect group $Q$. Then there exists a block $\beta$ of $Q C_{G}(Q)$ with defect group $Q$ satisfying $\beta^{H}=b$ and a unique block $\alpha(b)$ of $N_{G}(Q)$ such that $\alpha(b)=\beta^{N^{(}(Q)}$ and $\alpha(b)^{G}=b^{G}$. If $\alpha(b)$ is the principal block of $N_{G}(Q)$ then $b$ is the principal block of $H$.

Proof. By 1 (c) there exists a $\beta$ as required. If $\beta^{\prime}$ is another block of $Q C_{\theta}(Q)$ with $\beta^{\prime H}=b$ then $\beta^{\prime N_{B}(Q)}=\beta^{N_{B}(Q)}$ by Brauer's first main theorem, hence certainly $\beta^{N} G^{(Q)}=\beta^{\prime N_{G}^{(Q)}}$. So $\alpha(b)$ is well defined and $\alpha(b)^{G}=\beta^{G}=$ $\left(\beta^{H}\right)^{G}=b^{G}$. Finally, if $\alpha(b)$ is the principal block of $N_{G}(Q)$ then $\beta_{0}^{N_{G}(Q)}=\alpha(b)$, where $\beta_{0}$ is the principal block of $Q C(Q)$, by lemma $2(\mathrm{a})$. Hence $\beta_{0}=\beta^{g}$ for some $g \in G$ by $1(\mathrm{~d})$. Consequently $\beta=\beta_{0}$ by Lemma $2(\mathrm{~b})$, hence $b=\beta^{H}$ is the principal block of $H$ by Lemma $2(a)$, as required.

Brauer's thrid main theorem [1, 65.4]

Let $b$ be an admissible block of $H$. Then $b^{a}$ is the principal block of $G$ if and only if $b$ is the principal block of $H$. 
REMARK. In view Lemma $2(\mathrm{a})$ we have to show that if $b^{G}$ is the principal block of $G$ then $b$ is necessarily the principal block of $H$. We proof this below.

Proof. Let $b$ be an admissible block of $H$ with a defect group $Q$ and let $P$ be a Sylow $p$-subgroup $G$ which contains $Q$. We prove the theorem by induction on $|P: Q|$. Assume $P=Q$. Then $\alpha(b)$ of Lemma $2(c)$ must have defect group $P$, by $1(\mathrm{~b})$ and Lemma $2(\mathrm{a})$. On the other hand if $f$ is the principal block of $N_{G}(Q)$ then $f^{G}=B_{0}$ by Lemma $2(\mathrm{a})$ and $f$ also has defect group $Q$ by $1(\mathrm{~b})$. But then $\alpha(b)$ is the principal block of $N_{G}(Q)$, hence $b$ is the principal block of $H$, by Lemma $2(\mathrm{c})$, as required. Assume now that $|P: Q|>1$. Then $\alpha(b)$ has defect group $>Q$ by Brauer's first main theorem and $1(\mathrm{~b})$. Since $\alpha(b)$ is an admissible block of $N_{G}(Q), \alpha(b)$ is the principal block of $N_{G}(Q)$ by the induction hypothesis. But then $b$ is the principal block of $H$ by Lemma 2 (c) and the theorem is proved.

REMARK. No restriction on the field $K$ is really needed, for the above proof can be adopted to a purely module-theoretic context.

\title{
References
}

[1] L. DoRnhofF: Group Representation Theory, Part B, Marsel Dekker, N. Y. 1972.

[2] G. MichleR: Blocks and centres of group-algebras; Lecture Notes in Math. Springer, Berlin, 429-563.

\author{
Department of Theoretical Mathematics \\ The Weizmann Institute of Science \\ Rehovot, Israel
}

